\title{
PROFILOGRAPHY AND ANALYSIS OF SURFACE STRUCTURES OF MATERIALS
}

\author{
Gunnar Kunzel, Monika Hromasova, Miloslav Linda \\ Czech University of Life Sciences Prague \\ hromasova@tf.czu.cz, linda@tf.czu.cz
}

\begin{abstract}
Lifetime and operational reliability extension of engineering components is subject to high quality of their surface structure, i.e. dimensional accuracy and surface roughness. Measurement and evaluation of surface quality is also important in electrotechnics in case of ceramic carriers of thin layers. The resulting characterization of the surface should include prediction of some parameters of thin layers deposited on it. Increasing demands on quality parallel to a wide use of new construction materials (often with worsened machinability), the development of cutting materials and machine tools lead to requirements for appropriate measuring devices and evaluation methods. Profilography is an instrumental method of graphical representation of surface irregularities of the sample material by profilograph. Typically, the instrument uses a diamond needle attached to an electromagnetic sensor (devices produced by Taylor Hobson). The electrical output of the instrument is amplified and recorded. The aim of the paper is advancement in surface structure measurement principles and evaluation methods. The surface parameters are defined using geometrical and mathematical relations derived from current standards. 2D and 3D analysis is applied on particular examples. Some parameters are modelled using Scilab Xcos. Future developments in surface structure analysis and evaluation methods are discussed. Spatial 3D surface analysis allows to assess functional properties such as wear, friction, lubrication, fatigue properties etc., which are not accessible in 2D.
\end{abstract}

Keywords: profilography, measurement, evaluation of surface roughness.

\section{Introduction}

Profilography is an instrumental method of imaging the surface roughness of the sample material. The result is a repetitive graphic record of so-called profilograph. The most common way of obtaining information about the surface roughness is monitoring the surface with the needle tip. Diamond-tipped needle is flexibly pressed onto the surface, it is forced to move and tracks the surface roughness. The contact force is so small that there is no destruction, but it is sufficient enough to enable the tip to track the unevenness (surface roughness of the sample). At the output of the profilograph is usually socalled profilograph, i.e. a record in the form of continuous random curve, at present, usually in the form of a digital sequence of individual values.

A classical instrument is the profilograph from an English company Taylor Hobson with an electromagnetic sensor [1]. After amplification of the electric signal, there is a graphic record of the curve at the output of the profilograph, which is corresponding to the surface micro-roughness on defined length of the sample, alternatively a corresponding sequence of values which can be now stored in digital memory and other evaluations be done by using common software [2-4].

The aim pf the paper is advancement in surface structure measurement principles and evaluation methods. The surface parameters are defined using geometrical and mathematical relations derived from current standards. 2D and 3D analysis is applied on particular examples. Selected mathematical parameters are modelled using Scilab Xcos [5].

The Taylor Hobson company is the undisputed leading manufacturer of measurement systems for a surface structure analysis of material samples with a wide range of contact and non-contact sensors, and software for a complex evaluation of roughness, waviness and a basic profile of the material surface after any technological treatment.

The parameters of a spatial evaluation of the surface texture are not yet fully normalized. Therefore the company Taylor Hobson is co-working on a solution for a program that will lead to the creation of ISO standard. The new software module Talymap 3D Topography is processing a spatial characteristic from the data obtained by measuring a series of parallel surface profiles. For the evaluation of 3D it uses amplitude parameters (most are derived from 2D), further parameters describing the ratio of the surface material (ISO 13565 parameters related to the distribution of unevennesses, e.g. density of protrusions, developed surface), and finally the volume parameters (to quantify the ratio of the material volume or cavity) [6]. In addition, this program allows us to remove one profile of the evaluated surface and perform its evaluation in 2D [7-10]. 


\section{Materials and methods}

The examined objects are selected samples of materials with different technologies of surface treatment. Apart from this measurement and evaluation of the micro-roughness it is also important in electrotechnology for the ceramic pads under thin layers (e.g., resistive layers). Here not only a numerical determination of the pad character is important, but also the estimation of some important properties of the layer, which will be applied onto the surface. Table 1 shows the samples of selected materials.

Table 1

Samples of selected materials

\begin{tabular}{|c|c|c|c|c|}
\hline Number & Type & Profilograph & $\begin{array}{l}\text { Dimensions } \\
\quad(\mathbf{m m})\end{array}$ & $\begin{array}{l}\text { Max. deviation } \\
\text { from the profile } \\
\text { centre line }(\mu \mathrm{m})\end{array}$ \\
\hline 1 & $\begin{array}{l}\text { Pad made of molten } \\
\text { toglass eutal }\end{array}$ & $\begin{array}{l}\text { Almost ideal smooth } \\
\text { surface }\end{array}$ & $60 \times 60$ & 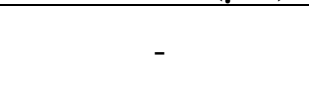 \\
\hline 2 & $\begin{array}{l}\text { Glazed glass-ceramic } \\
\text { sitall }\end{array}$ & $\begin{array}{l}\text { Smooth, slightly } \\
\text { waved surface }\end{array}$ & $60 \times 48$ & 0.5 \\
\hline 3 & Pad corundum & $\begin{array}{l}\text { Better readability of } \\
\text { the surface }\end{array}$ & $12 \times 23$ & 3 \\
\hline 4 & $\begin{array}{l}\text { Rod corundum with } \\
\text { grinded and fused } \\
\text { surface }\end{array}$ & $\begin{array}{c}\text { Significant } \\
\text { unevenness of the } \\
\text { surface }\end{array}$ & $\phi 3 \times 9$ & 2 \\
\hline 5 & $\begin{array}{l}\text { Rod corundum with } \\
\text { grinded and fused } \\
\text { surface }\end{array}$ & Resilient roughness & $\phi 6.5 \times 11.6$ & to 2 , sporadically 3 \\
\hline 6 & $\begin{array}{l}\text { Rod corundum rough, } \\
\text { ungirded surface }\end{array}$ & $\begin{array}{l}\text { Better readability of } \\
\text { the surface }\end{array}$ & $\phi 4 \times 12$ & 3 - 4 permanently \\
\hline
\end{tabular}

To display surface roughness, the classical profilograph uses an assumption that the measuring tip is conducting a movement of two segments, i.e. forcibly moves in a uniform motion in a plane perpendicular to the surface of the examined surface and follows the surface unevenness, and it causes the movement in a direction perpendicular to the surface (axis of the needle remains in a plane perpendicular to the surface).

The final track of the composite movement is a plane curve which is identical with the penetration of considered perpendicular plane to the actual surface area. The curve must be placed in the coordinate system. The y-axis corresponds to the distance of individual surface points from the surface level. The x-axis corresponds to the first forced segment of the movement and it is put to the surface level. Intersection is a graph of the function $y=f(x)$ and it represents a profile of the surface sample. However, instead of the surface level, only the $\mathrm{x}$-axis is determined, which is placed within, and this is because of a condition of a zero mean value at the sampling interval (e.g., at the length $L$ of $1 \mathrm{~mm}$ ) (1)

$$
\frac{1}{x_{2}-x_{1}} \int_{x_{1}}^{x_{2}} f(x) d x=0
$$

The sampling interval is a field of a profile definition and an integration interval. At the output of the profilograph there is a plotter that records the progress $F(\xi)$, which is generally a linear display of the profile $f(x)$ with the transformation equations $(2,3)$

$$
\begin{gathered}
\xi=m_{x} x, \\
\eta=F(\xi)=m_{y} f(x),
\end{gathered}
$$

Profilographs allow visual and dimensional comparison of profiles. For workpieces in mechanical engineering it may be an effective method of controlling the technological process and the quality of a surface finish, e.g., by machining, where the profiles are periodic. The assessment of the profile angles 
on profilographs is not easy. A relation of an angle $\psi$ on the profilograph and of an angle on the profile of the actual surface is non-linear, if equation (4)

$$
\alpha=\operatorname{arctg}\left(\frac{m_{x}}{m_{y}} \operatorname{tg} \psi\right)
$$

Therefore, a transformation protractor was constructed for each pair of scales $m_{x}, m_{y}$. The protractor shows locations for the instrument's micro-tip, where the tip is not following the surface steeper than $45^{\circ}$ but slides on the surface with its side. A profilographic evaluation of the surface roughness is carried out according to relevant standards. In the original Czech Norm ČSN 014450 the evaluating parameters $R_{z}, R_{a}, R_{\max }, R_{\min }$ were stated [6].

The profilograph evalution is in electrotechnology completely inadequate for the pads under thin layers. The outcome of the evaluation should be a numerical determination of the pad surface character and also the prediction of important properties of the applied layer. Therefore, the so-called analytic profilographic evaluation of the surface roughness has been implemented based on the operations of mathematical analysis of differentiation and integration. The fundamental difference is that the evaluation in this case includes every point of the profile. The simplest method of profilograph analysis is based on measuring the length of the curve defined by the equation (5).

$$
l=\int_{x_{1}}^{x_{2}} \sqrt{1+\left[f^{\prime}(x)^{2}\right] d x},
$$

At present, it is best to leave this operation to the PC instead of the former mechanical bypassing and measuring the length or the model on so-called analogue computers.

The value of the arithmetical mean deviation $R_{a}$ from the profile centre line can be defined using integral (6) where $L=B-A$.

$$
R_{a}=\frac{1}{L} \int_{A}^{B}|f(x)| d x,
$$

It would be possible to similarly define the mean quadratic length from the profile centre line. However, it is a parameter (analogous to the effective value of alternating current) defined formally only mathematically, i.e. without a reference to any particular physical, technological or functional surface characteristic about which cannot be stated anything specific. It is important for a surface evaluation of the workpiece that the profile is in transverse direction to the machinging trails. In (6) there is an evaluation of the workpiece profiles suggested as a ratio of two integrals, which is the ratio $R_{a}$ to the mean value of the profile derivative (7)

$$
\lambda_{a}=2 \pi \frac{\int_{0}^{L}|f(x)| d x}{\int_{0}^{L}\left|f^{\prime}(x)\right| d x},
$$

where $\lambda_{a}$ - mean wavelength of the frequency segments of the Fourier spectrum of the profile.

$\lambda_{a}$ is dependent on the power spectral density of the profile. Today's options of the profilograph analysis are mainly determined by the current technical quality of profilographs, and by the software, which enables the evaluation of the roughness in $2 \mathrm{D}$ and $3 \mathrm{D}$ according to the updated ISO standards (ISO 13565-2).

\section{Results and discussion}

After a profilograph calibration, the classic method of profilography assumes the profile unevenness recording on a certain length of the sample in the x-direction that corresponds with the middle line $\mathrm{m}$ (6). As per Tab. 1, where the measured profilograms were evaluated. A total of 10 measurements were made for five parallel locations of the surface for each sample, and the values 
were averaged (Tab. 2). The measured profilogram pad of corundum is in Fig. 1. Each profile was evaluated by the parameters defined by the equations $(5,6,7)$.

For these calculations we created a block diagram, the input pf which is a corresponding profilogram for each measured profile and at the output are the evaluating parameters $R_{a}, 1, \lambda_{a}$. The calculation is conducted in the Xcos (Fig. 2).

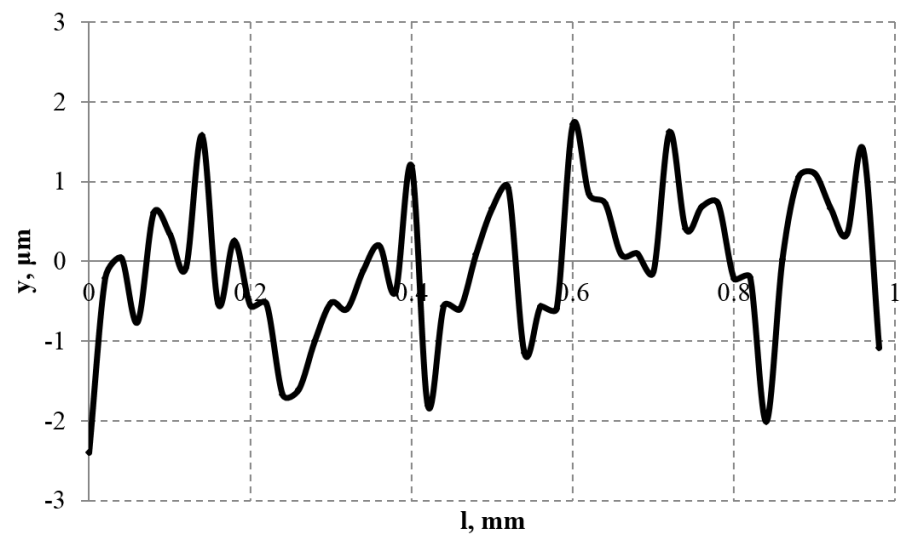

Fig. 1. Profilograph pad of corundum

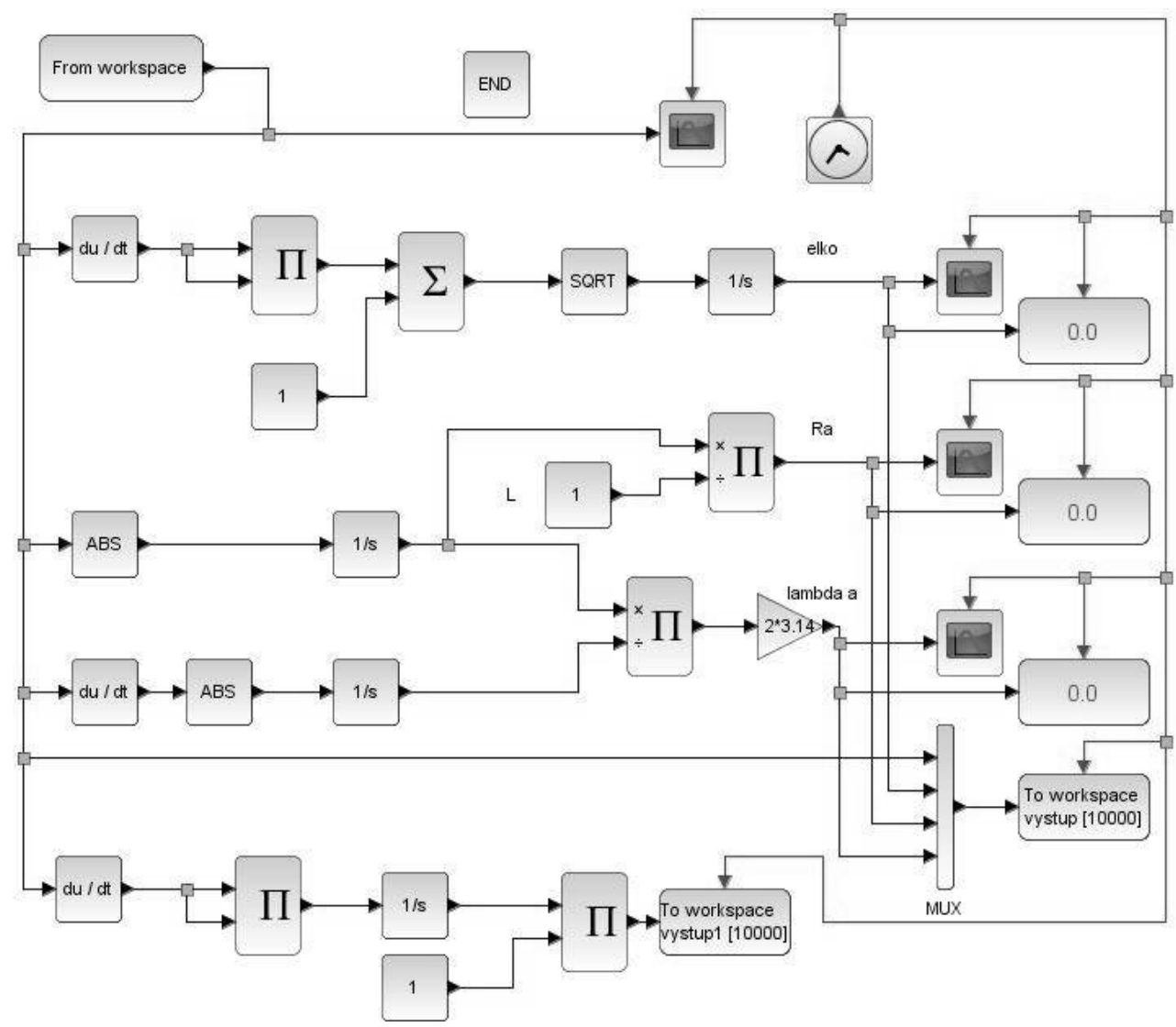

Fig. 2. Block diagram in Scilab XCos

Table 2

Averaged values for all samples

\begin{tabular}{|c|c|c|c|c|c|c|}
\hline Samples & $\mathbf{1}$ & $\mathbf{2}$ & $\mathbf{3}$ & $\mathbf{4}$ & $\mathbf{5}$ & $\mathbf{6}$ \\
\hline$R_{a}$ & 0.7 & 0.8 & 0.7 & 0.7 & 0.7 & 0.5 \\
\hline$l$ & 80.3 & 78.4 & 69.9 & 82.1 & 75.6 & 76.1 \\
\hline$\lambda_{a}$ & 49.5 & 67.6 & 63.1 & 38 & 47.6 & 19.7 \\
\hline
\end{tabular}


The conducted analytic profilographic evaluation of the pad surface for thin layers leads to the hypothesis that there is a functional dependence between the parameters of the surface roughness obtained by profilograph analysis and the variable corresponding to the surface function. The hypothesis is confirmed during investigating the functional dependence of the sheet resistance of the thin conductive layer, vapour-deposited in vacuum on the pad surface, on the functional parameter $K_{R}$ unevenness of this pad. The assumption is that the resistive layer is a compact metal layer with an electrical conductivity equal in all directions, which monitors its upper and lower area of the surface roughness on the ceramic pad surface. The thickness of the deposited layer is $2.10^{-7} \mathrm{~m}$. The pad profile assumes a continuity and stationarity of a random function $f(x)$ of the profile. In case of a periodic waveform, the period must be sufficiently short for the sampling interval. Then applies (8)

$$
K_{R}=\frac{R}{R_{0}}=1+\frac{1}{x_{2}-x_{1}} \int_{x_{1}}^{x_{2}}\left[f^{\prime}(x)^{2}\right] d x,
$$

where $K_{R}$ - functional parameter of the layer;

$R$ - layer resistance on the pad with a profile $f(x), \Omega$;

$R_{0}$-layer resistance on the perfectly straight pad with profile $f(x)=$ const., $\Omega$;

$x_{1}, x_{2}-$ starting and ending point of the sampling interval $\left(x_{2}-x_{1}=1.10^{-3} \mathrm{~m}\right), \mathrm{m}$.

The electrical signal proportional to the parameter $K_{R}$ arises from the measured profilogram $f(x)$ and it is transformed to the output signal $K_{M}$ according to (8). For the imaging scale $k_{1}$ applies (9)

$$
K_{M}=k_{1} \int_{x_{1}}^{x_{2}}\left[f^{\prime}(x)^{2}\right] d x .
$$

The theoretical dependence is verified (10)

$$
\frac{R}{R_{0}}-1=\frac{K_{M}}{k_{1}} \frac{1}{x_{2}-x_{1}},
$$

of which is the graph of increasing line passing through the origin. During the auxiliary variable $\frac{R}{R_{0}}-1$ regression for $K_{M}$ for the function $y=k x$ is the slope of the straight line given by the equation (11)

$$
k=\frac{k_{2}}{k_{1}\left(x_{2}-x_{1}\right)},
$$

where another constant corrects the slope of the straight line for a particular set of samples.

Prediction of the sheet resistance is then based on the equality (12)

$$
K_{R}=k K_{M}+1,
$$

Regression for a condition of least squares deviations is given by relation (13)

$$
k=\frac{\sum_{i=1}^{n} K_{M i}\left(\frac{R}{R_{0}}-1\right)_{i}}{\sum_{i=1}^{n} K_{M i}^{2}},
$$

where $n=50$ for each sample.

Evaluation of 300 values from 6 samples in total provides an interval of $1.23<k<1.44$ (Fig. 3). The measurements of the resistance $R$ of the layer were done using a double-tipped probe. Resistance $R_{0}$ is a resistance of the vapor-deposited layer on glass eutal, which surface is smooth.

The slope of the straight line $k=0.9$ is for samples 1 and $2, k=1.23$ is for samples 4 and 5 and $k=1.44$ is for samples 3 and 6 . 


\section{Results and discussion}

The characteristic of the surface structure, including the definition of parameters and their measurements, is part of the standards. Standardized description of the structure includes roughness, waviness and primary profile. At present, the basis for the evaluation of the surface structure remains the method of profilography. The obtained digitised profile of the surface is processed and evaluated using a variety of standardized and non-standardized height-, length- and shape-parameters. The processing of measured data is conducted either directly in the evaluation part of the profilograph or using the supplied software on PC. Perspectives of the profilograph analysis, mainly in electrotechnology, however lie in the formulation of correlative and functional dependence between the parameters of unevenness and functional properties of the surface.

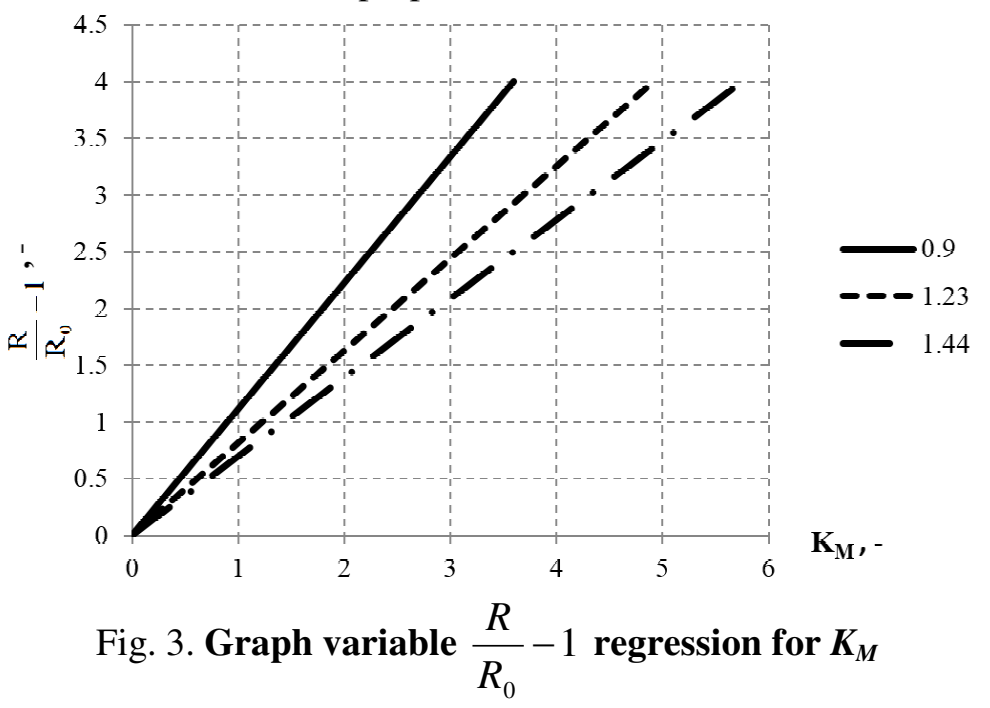

Therefore, the functional dependency of the sheet resistance of the thin conductive layer vapourdeposited onto the ceramic pad surface was designed and verified by the new method. Other options of evaluation are provided using a spatial analysis of the surface (3D) when assessing the functional properties. The method is new and the dependence was proved, but some deviations during the first experimental verification have not been satisfactorily explained. For further research, it may be necessary to refine the measurement method of measuring the square resistance using a four-tipped probe and it will be necessary to measure the statistical distributions of $K_{M}$ on the pad surface in different directions.

\section{References}

1. Operator's Handbook for Talystep. Rank Taylor Hobson, 1982.

2. Chiffre L. De, Lonardo P., Trumpold H., Lucca D. A., Goch G., Brown C. A., Raja J., Hansen H. N. Quantitative Characterisation of Surface Texture. CIRP Ann. - Manuf. Technol., vol. 49, no. 2, 2000, pp. 635-652.

3. Whitehouse D. J. Surface metrology. Meas. Sci. Technol., vol. 8, no. 9, 1997, pp. 955-972.

4. Whitehouse D. J. Handbook of Surface Metrology. CRC Press, 1994.

5. Scilab, Enterprises, and S.A.S, "Xcos for very beginners tutorial." [Online] [28.01.2017] Available at: https://www.scilab.org/content/view/full/957.

6. ISO Standard, C CSN EN ISO 4287 "Geometrical product specifications (GPS) - Surface texture: Profile method - Terms, definitions and surface texture parameters"(In Czech).

7. HOMMELWERKE GmbH, Ed., Functional Description User Interface TURBO WAVE. V7.1. [Online] [28.01.2017] Available at: pgikorea.kr/board/data/product/50/10014025_TURBO_WAVE_Englisch.pdf

8. Piska M., Metelkova J. On the comparison of contact and non-contact evaluations of a machined surface. MM Sci. J., vol. 7, 2014, pp. 1-5.

9. Taylor Hobson Precision, Taly Map Software. [Online] [28.01.2017] Available at: http://www.taylorhobson.com.br/pdf/113_espec.pdf

10. Veeco, Technology and products. [Online] [28.01.2017] Available at: http://www.veeco.com/technologies-and-products. 